\title{
CALIDAD DEL AMBIENTE Y CALIDAD DE LA CONDUCTA SEGÚN ALUMNOS DE TERCER CICLO DE DOS COLEGIOS PRIVADOS SALVADOREÑOS
}

\author{
QUALITY OF ENVIRONMENT AND QUALITY OF \\ BEHAVIOR ACCORDING TO STUDENTS FROM TWO \\ SALVADORAN PRIVATE MIDDLE SCHOOLS
}

\author{
Henrry Ademir García Figueroa \\ Colegio Adventista John Nevins Andrews, El Salvador \\ henrryxade@hotmail.com \\ https://orcid.org/0000-0002-4823-0505
}

\begin{abstract}
RESUMEN
Esta investigación, de abordaje cuantitativo y diseño descriptivo y correlacional, analizó la relación entre la percepción del grado de calidad del ambiente escolar, familiar, social y religioso y el grado de calidad de la conducta autopercibida de 158 estudiantes de tercer ciclo de educación básica de dos colegios privados salvadoreños. Los resultados del estudio muestran que la calidad percibida de los ambientes sociales, familiares, religiosos y escolares es predictora significativa de la calidad de la conducta autopercibida. Se encontró también que en el caso de las mujeres los predictores más significativos fueron el ambiente religioso y el ambiente social, mientras que en los hombres los principales ambientes predictores de la conducta fueron los ambientes familiar y social.
\end{abstract}

Palabras clave: ambiente escolar, ambiente familiar, ambiente social, ambiente religioso, calidad de la conducta

\section{ABSTRACT}

This research, with a quantitative approach and descriptive and correlational design, analyzed the relationship between the perception of the degree of quality of the school, family, social and religious environment and the degree of quality of the self-perceived behavior of 158 students of the third cycle of basic education from two private Salvadoran schools. The results of the study show that the perceived quality of social, family, religious and school environments is a significant predictor of the quality of self-perceived behavior. It was also found that in the case of women the most significant predictors were the religious environment and the social environment, while in men the main predictive environments of behavior were the family and social environments.

Keywords: school environment, family environment, social environment, religious environment, quality of behavior 


\section{Introducción}

Dos investigaciones desarrolladas en Centroamérica (Cerna Vanegas y Meza Escobar, 2009; Jesse Rock, 2011) revelaron datos significativos acerca de la influencia que tienen los factores ambientales sobre la conducta de los alumnos y cuáles de ellos poseen un grado de influencia mayor. Ambos estudios coinciden en que las dos dimensiones que se correlacionan en mayor grado con la conducta de los estudiantes son el ambiente familiar y el ambiente religioso.

Este estudio pretende analizar los factores ambientales predictores de la conducta según la percepción de los alumnos. Se considera importante determinar el factor ambiental con mayor grado de incidencia sobre la conducta autopercibida de los estudiantes en los colegios del estudio.

Con el propósito de sustentar la investigación, se presentan a continuación los conceptos de ambiente familiar, ambiente social, ambiente escolar, ambiente religioso, y la relación entre ambiente y conducta.

\section{Ambiente familiar}

La familia es la primera fuente de aprendizaje y conocimiento con la que el niño interactúa (Bustos Correa et al., 2001). El ambiente familiar tiene varias dimensiones: el control, la cohesión, el conflicto, la vida social y la recreación para el adolescente (Cantón-Cortés et al., 2010). Un buen ambiente familiar constituye un sistema para amortiguar el estrés ante experiencias traumáticas como el divorcio de los padres o el abuso sexual infantil y contribuye a reducir el comportamiento infantil agresivo.

La importancia de que el niño sea tratado bien en su hogar es de vital importancia. Según Rivero y Martí-
nez-Pampliega (2010), la familia desarrolla patrones de comunicación en la dimensión de conversación, que incluye la expresión de ideas y aceptación de diferencias, y en la dimensión de conformidad, que incluye la obediencia y el rechazo de la diferencia, por lo que una familia que tiene estabilidad en sus patrones de comunicación puede ser predecible incluso si existe maltrato que repercute en el niño.

Según Bachman (2007), en los adolescentes un clima conflictivo entre los padres constituye un predictor negativo de su conducta y disminuye sus habilidades sociales.

En ese sentido, otro estudio sobre conflictos interparentales correlacionado con la aparición de problemas conductuales en adolescentes que pertenecían a familias intactas y monoparentales (Mayorga Muñoz et al., 2016) observó que el divorcio es la pérdida de un punto de referencia vital, como ver a sus padres a diario bajo el mismo techo y compartiendo alimentos y que el infante reciba atenciones en todo momento, siendo sustituido por visitas y cuotas alimenticias impersonales, situación en la que el niño continúa una vida de conflictos que afecta su conducta interna y externa.

El ambiente familiar con "disciplina severa", que manifiesta hostilidad y rechazo, también es un predictor de conducta antisocial. En contraposición, la comunicación favorece la disciplina evitando la conducta antisocial (Quiroz del Valle et al., 2007). Esa comunicación confronta desafíos ante el ocio digital que altera las dimensiones de cohesión, flexibilidad y funcionamiento familiar, según Valdemoros-San-Emeterio et al. (2017), quienes sostienen además que un menor consumo digital en los hijos se vincula a familias que gozan de una 
mayor fortaleza en la vinculación emocional entre sus miembros, en la reciprocidad emocional que se da o puede darse entre los componentes de la familia, en la implicación familiar, en el respeto mutuo entre padres e hijos, así como en el establecimiento de "fronteras internas" y alianzas que se establecen en las relaciones intergeneracionales.

De acuerdo con Moreno Ruiz et al. (2009), la autoridad de los padres constituye un "marco de referencia de los adolescentes para actuar bajo el control de autoridad formal del profesor" ( $p$. 132).

Pitsoane y Gasa (2018) encuentran que el apego deficiente padres-hijos constituye un obstáculo mayor para un desarrollo que asegure la estabilidad emocional y, por lo tanto, un comportamiento correcto.

\section{Ambiente social}

Jiménez et al. (2014) se refieren al ambiente comunitario que conlleva relaciones familiares, percepción del ambiente comunitario y actitud hacia la autoridad e instituciones. La actitud correcta y el bienestar subjetivo del adolescente reducen la probabilidad de agresión manifiesta hacia sus pares.

Muy similar al caso salvadoreño es el caso brasileño de las favelas, donde se encuentran comunidades en condiciones de desamparo. Dias Vieira y AbuJamra Zornig (2015) sostienen que, en esos casos, la comunidad es el colectivo que auxilia al sujeto en el proceso de elaboración del potencial traumático del ambiente en que vive, con un efecto antitraumático, donde maduran precozmente los adolescentes para que cobre sentido la existencia.

Los adolescentes son capaces de alcanzar competencias sociales, pero cuan- do carecen de ellas su comportamiento es antisocial, de dos maneras: delictivo y no delictivo. Arce et al. (2011) afirman que los menores con comportamientos delictivos o antisociales tienen incompetencia social y que, a mayor grado de incompetencia social, se observa mayor nivel de gravedad del comportamiento desviado. Por su parte, Johnson et al. (2012), al estudiar el grado de violencia en los centros escolares urbanos, señalan que "el ambiente escolar sin seguridad escolar tiene lugar por el ambiente del vecindario, por lo que describir violencia solo en la escuela no es un énfasis suficiente" (p. 99).

Esto tiene sentido en el contexto salvadoreño al analizar que ingresan a los centros escolares adolescentes de vecindarios peligrosos pertenecientes a grupos delincuenciales que establecen relaciones interpersonales con el personal de apoyo, docentes y demás alumnos. Con ello, exponen a la escuela a manifestaciones de violencia y conducta antisocial que llega a deteriorar tales relaciones, derivando en conflictos y actos delictivos.

\section{Ambiente escolar}

Es en el ambiente social donde el adolescente debe adquirir alguna formación. Según Papšová et al. (2012), existe relación entre "el incremento de satisfacción escolar con la percepción del clima de aula social positivo" (p. 134). Un ambiente negativo puede generarse por parte del profesor en el aula cuando manifiesta enojo, falta de amabilidad, cortesía, consideración y respeto por los alumnos. Igualmente, las normas quebrantadas producen insatisfacción con el centro educativo, generando abandono o dejando lugar a conductas antisociales. El análisis del ambiente escolar 
debe incluir la discriminación que algunos alumnos reciben por parte de sus compañeros en el aula y que afectan sus relaciones sociales. Las relaciones interpersonales son muy importantes para los adolescentes.

Los adolescentes necesitan las mejores medidas que los adultos puedan brindar en su atención emocional. Karimi Koma (2014) estudió el desarrollo social de jóvenes bajo la influencia de directores de escuela con especialidad en administración y observó que, cuando los estudiantes se hallan bajo el trabajo especializado de un director capacitado en área administrativa, los docentes tienen mejores procesos disciplinarios y los alumnos adquieren la conducta social que desea la institución educativa, lo que supone una inversión en capacitación de personal, para adquirir mayor calidad, profesionalidad y prestigio, por lo que es indispensable la capacitación periódica de docentes y de administradores, y que esa conducta interpersonal con los alumnos sea objeto de evaluación docente periódica.

De acuerdo con la Real Academia de la Lengua Española (2014, citada en Carranza et al., 2017), el ambiente escolar está conformado por los "escenarios posibles de aprendizaje con matices de la clase con alumnos y el tipo de propuestas del docente" (p. 2).

Conservar un ambiente escolar positivo es un gran desafío que conlleva la adquisición de experiencia en el aula para conservar disciplina y al mismo tiempo el desarrollo afectivo, pues el clima de la clase óptimo para aprendizaje es el que evita la violencia y favorece la aceptación y el reconocimiento, motivando a seguir normas de convivencia grupal pacífica. Cuando hay violencia el clima se torna negativo para el proceso de enseñanza-aprendizaje, distorsionando las relaciones alumno-profesor (Infante et al., 2003).

Para mejorar el ambiente escolar, Boarini (2013) llama a la administración escolar a tener apertura a la tecnología y al docente a desarrollar clases centradas en el alumno. Al mismo tiempo, la escuela debe ser una extensión de la familia. La disciplina debe ser un tema y un esfuerzo colectivo.

Foà et al. (2012) atribuyen la aparición de comportamientos agresivos al reflejo de una "distribución contextual de las normas sociales, con dos tipos de conducta, transgresora y agresora, que pueden ser prevenidas si se conduce a los jóvenes con una actitud positiva para someterse a ellas, otorgando legitimidad a la autoridad escolar" (p. 17). La conducta agresora excede las normas sociales, criterios morales e institucionales de control social a sabiendas, desafiando a la autoridad e incitando la expresión de violencia injustificada y tendiente al mal.

Según Guerra Vio et al. (2012), el clima social escolar, que comprende más que la infraestructura ordenada, segura y limpia, está referido "a la percepción de los estudiantes de ser tratados justamente por sus profesores, de comprometerse en las tareas escolares y de ser reconocidos por ello" (p. 113).

\section{Ambiente religioso}

Pillon et al. (2011) observaron que los estudiantes consideraban la espiritualidad como un tema central para la vitalidad de su estilo de vida, entendiéndose como "una alternativa poderosa para construir sentidos que permiten a la persona dar significado a la vida" ( $p$. 102).

La religión profesada y los comportamientos religiosos tienen impacto 
sobre la salud, el desarrollo mental y social de los niños que trasciende al simple acto de asistir a servicios religiosos (Schottenbauer et al., 2007).

La base de esta dimensión religiosa se encuentra bajo la responsabilidad de los padres. Un estudio (Petts, 2011) concluyó que los "padres tienden a tener mayor religiosidad después del nacimiento de un hijo y, durante su edad temprana, la religiosidad de las madres reduce problemas de conducta" (p. 187). Fortalecen el estilo de vida religioso y permiten al joven tener identidad y pertenencia. Así mismo, los padres orientan a sus hijos no solo con conocimientos sobre Dios, sino con prácticas que se convierten en hábitos y desarrollan el carácter.

La Biblia y la justicia como dimensiones religiosas son factores protectores ante la disposición a conductas antisociales y predictores de la conducta prosocial (Grier y Gudiel, 2011).

Un estudio de Driskell y Lyon (2011) mostró que las creencias religiosas afectan tanto a los "comportamientos seculares como los espirituales, justificando sus comportamientos complejos con un fundamento lógico en sus creencias" ( $p$. 400), lo que implica que afectan su toma de decisiones, su esperanza, su cosmovisión y su conducta.

Las escuelas patrocinadas por la iglesia muestran seis áreas de desarrollo de la vida espiritual: mejor autoestima, mayor rechazo de drogas, menor apoyo a comportamientos ilegales, menor actitud racista, mayor creencia cristiana y perspectiva moral y sexual conservadora (Francis et al., 2014).

\section{Ambiente y conducta}

Para Slattery y Meyers (2014), los problemas de conducta tienen su origen en la exposición a una "comunidad violenta como el más poderoso predictor de la conducta antisocial, y el control parental es débil factor predictor para la conducta antisocial, contrario a lo que sostenían investigaciones previas" (p. 51). En la realidad salvadoreña se ha manifestado plenamente que la comunidad violenta lleva a los jóvenes a integrarse en estructuras antisociales.

En el contexto de violencia del ambiente social y con el estilo de crianza "simplemente autoritario o autoritario materno" se tienen predictores de trastorno infantiles del comportamiento por las limitaciones sociales que propone ese estilo de crianza, la reducción de capacidades para asimilar nuevos contextos sociales, la aversión hacia la autoridad sin ninguna motivación extrínseca de los padres e incluso causando más complicaciones que una crianza permisiva sin disciplina (Loona y Kamal, 2012).

La presente investigación tuvo como objetivo principal conocer si el grado de calidad del ambiente percibido (escolar, familiar, social y religioso) influye en el grado de calidad de la conducta de los estudiantes de dos colegios privados en El Salvador. Este estudio sirve para analizar la complejidad de un fenómeno social que merece ser observado desde la mayor cantidad de perspectivas posibles, pues atañe a cada sector de la comunidad educativa: padres, educadores y alumnos, poniendo de manifiesto las tendencias y orientaciones supeditadas a diversos factores vitales en la toma de decisiones de los alumnos de los colegios estudiados, en donde la construcción del carácter tiene lugar en su formación y se consolida al mismo tiempo un sistema educativo que optimice el valor agregado de los principios bíblicos. 
A su vez tal conducta y su tendencia por parte de los estudiantes se considera en el siglo XXI como un desafío familiar e institucional enorme para la educación y su eficacia en esta región.

\section{Metodología \\ Tipo de investigación}

Esta investigación utilizó un abordaje cuantitativo y un diseño descriptivo y correlacional.

\section{Participantes}

Participaron del estudio 158 alumnos de tercer ciclo de dos colegios privados de El Salvador, agrupados como se detalla en la Tabla 1.

\section{Tabla 1}

Distribución de los participantes por colegio

\begin{tabular}{lcc}
\hline \multicolumn{1}{c}{ Institución } & $n$ & $\%$ \\
\hline Colegio 1 & 93 & 58.86 \\
Colegio 2 & 65 & 41.14 \\
Total & 158 & 100.00 \\
\hline
\end{tabular}

\section{Instrumentos}

Percepción de la calidad del ambiente. Para medir el grado de la calidad del ambiente, se usó el instrumento genérico elaborado por Cerna Vanegas y Meza Escobar (2009), el cual consta de cuestionarios para medir la percepción de la calidad del ambiente en cuatro dimensiones: ambiente escolar, ambiente familiar, ambiente social y ambiente religioso. Para calcular la confiabilidad del instrumento, los autores utilizaron la prueba alfa de Cronbach, en donde la dimensión ambiente escolar obtuvo un índice de .823, para 13 ítems; la dimensión de ambiente social obtuvo un índice de .817, para 11 ítems; la dimensión ambiente familiar obtuvo un índice de .801, para 12 ítems; y la dimensión ambiente religioso obtuvo un índice de .907 , para 11 ítems. El índice del coeficiente alfa de Cronbach correspondiente a los 47 ítems del instrumento fue de .915 .

Calidad de la conducta autopercibida. El grado de calidad de la conducta autopercibida fue evaluado mediante un instrumento elaborado por Cerna Vanegas y Meza Escobar (2009) que consta de 38 ítems, con una valoración tipo Likert que va de uno a cinco, de la siguiente manera: (a) nunca, (b) casi nunca, (c) a veces, (d) casi siempre y (e) siempre. Para calcular la confiabilidad del instrumento, los autores utilizaron el coeficiente alfa de Cronbach, que mostró un índice de .900 para los 38 ítems del instrumento.

\section{Análisis de datos}

Para determinar la relación entre la percepción del grado de calidad del ambiente y la conducta autopercibida, se utilizó un análisis de regresión lineal múltiple. El análisis de varianza de un factor y la prueba $t$ para muestras independientes se usaron para observar el efecto de las variables demográficas sobre las variables del estudio.

\section{Resultados \\ Análisis descriptivo}

Para valorar los ambientes de los estudiantes se utilizaron escalas Likert de cinco puntos. En todos los casos, a mayor puntaje se interpreta que el estudiante percibía un mejor ambiente.

En la Tabla 2 se puede observar que la percepción del ambiente familiar y el religioso obtuvieron las puntuaciones más altas, mientras que los ambientes escolar y social las más bajas. Se observa más diferencia de opinión en el ambiente religioso, en cuya distribución se 
observa mayor asimetría, mientras que la de la percepción del ambiente escolar es más homogénea.

Respecto a los ítems de percepción del ambiente escolar, el aspecto mejor percibido es el que tiene que ver con las amistades y los programas espirituales, mientras que la peor percepción es sobre el vocabulario que usan los alumnos. En el ambiente familiar se destacan los elementos que tienen que ver con la limpieza, el trato de los padres, la alimentación y las relaciones familiares. Por otro lado, lo peor valorado tiene que ver con los libros que se leen. Respecto al ambiente social, resultan mejores los aspectos de respeto y amistad y resultan más bajos los aspectos de vocabulario y de interés por las actividades espirituales. Por último, en el ambiente religioso resaltan los aspectos de limpieza, respeto por autoridad, así como el trato a las personas, pero la conducta de los jóvenes obtuvo la puntuación más baja.

\section{Tabla 2}

Descriptivos de la calidad percibida de los ambientes estudiados

\begin{tabular}{lllcc}
\hline \multicolumn{1}{c}{ Ambiente } & $M$ & $D E$ & Asimetría & Curtosis \\
\hline Escolar & 3.8 & 0.575 & -0.365 & 0.080 \\
Familiar & 4.2 & 0.623 & -0.699 & 0.061 \\
Social & 3.6 & 0.755 & -0.428 & 0.135 \\
Religioso & 4.2 & 0.821 & -1.628 & 3.159 \\
\hline
\end{tabular}

La escala de conducta autopercibida de los estudiantes resultó con una media de $4.0(D E=0.478)$. Dado que la escala era de uno a cinco, el nivel de calidad de conducta general es del $75 \%$, donde un mayor valor se interpreta como mejor calidad.

Según las respuestas a los ítems, las mejores conductas incluyen respeto al director del colegio, bañarse diariamente, usar uniforme y respetar al sexo opuesto, las cuales dicen manifestar siempre. Las conductas que regularmente practican incluyen ver programas educativos en TV, escuchar música cristiana en casa, colaborar con vecinos, leer y jugar con vecinos. Además, los participantes señalaron que casi nunca entran a sitios prohibidos en internet.

\section{Análisis predictivo}

La percepción de la calidad de los ambientes es predictora significativa de la calidad de la conducta autopercibida $\left(F_{(3,154)}=23.891, p=.000\right)$. La varianza explicada es del $30.4 \%$ y resultan predictores significativos tres de los ambientes: el ambiente social $(\beta=.246, p$ $=.005)$, el ambiente familiar $(\beta=.215$, $p=.015)$ y el ambiente religioso $(\beta=$ $.202, p=.031)$. Por lo tanto, mientras mejores sean las percepciones de estos ambientes, la calidad de la conducta autopercibida será también mejor. De hecho, la percepción del ambiente escolar también es predictora significativa, solo que por la multicolinealidad no aparece en el modelo de regresión, pero su relación con la calidad de la conducta autopercibida es significativa $(r=.385, p=$ $.000)$.

En un análisis por género, en el caso de las mujeres, son predictoras significativas de la calidad de la conducta autopercibida $\left(F_{(2,80)}=14.368, p=.000\right.$, $\left.R^{2}=.246\right)$ la percepción del ambiente 
religioso $(\beta=.302, p=.015)$ y la del ambiente social $(\beta=.270, p=.030)$, en ese orden de importancia. En el caso de los hombres, son predictoras $\left(F_{(2,72)}=\right.$ $\left.25.857, p=.000, R^{2}=.402\right)$ la percepción del ambiente familiar $(\beta=.447, p=$ $.000)$ y la del ambiente social $(\beta=.289$, $p=.008)$, en ese orden de importancia.

Al realizar análisis comparativos, no se encontró diferencia significativa de los valores de las variables del estudio entre los estudiantes de las diferentes escuelas, ni entre los estudiantes agrupados según las personas con las cuales vivían. Pero la calidad de la conducta autopercibida es significativamente mejor $\left(t_{(156)}=3.072, p=.003\right)$ en los cristia$\operatorname{nos}(M=4.1, D E=0.478)$ que en los no cristianos $(M=3.9, D E=0.454)$.

\section{Discusión}

De las dimensiones para medir el grado de calidad del ambiente, tomando en cuenta la crisis del tejido social salvadoreño, siendo de los países que sufren mayor violencia del mundo, el ambiente social percibido por los estudiantes tiene un grado de influencia en la calidad de su conducta autopercibida. Sin embargo, en este estudio no es la dimensión del ambiente con mayor influencia, pues el ambiente familiar y el ambiente religioso percibidos son los que ejercen mayor grado de influencia sobre su conducta autopercibida.

Según la percepción de los varones, los ambientes predictores de su conducta fueron el familiar y el social, en ese orden. Esto tiene su interpretación en el contexto de la realidad nacional, pues las influencias que apartan de la moral e involucran a los estudiantes adolescentes en grupos criminales, degradando su condición moral, son las de sus pares, porque buscan en ellos el afecto que no reciben en su hogar, desintegrado por la migración ilegal de sus progenitores o por descuido de ellos, que van perdiendo su autoridad. Esto también es comprensible en el contexto de tener que vivir en sectores de riesgo de violencia, por lo que muchos padres y familiares encargados prefieren una educación cristiana para modelar valores para la vida, principios morales, laboriosidad y respeto a las autoridades.

Para la percepción de las niñas, los ambientes predictores de su conducta autopercibida fueron el religioso y el social, en ese orden. Esto reviste importancia para el sistema educativo, pues comprende la formación integral de los jóvenes, la preparación para el servicio y su capacidad de ajuste social fuerte ante la realidad nacional que deben afrontar. Perciben que los valores morales son necesarios y conservarlos es un elemento de su cosmovisión que les proporcionan una identidad. Algunos estudios previos han hecho énfasis sobre el desarrollo espiritual de los estudiantes para afrontar la vida.

Existen documentos internacionales sobre esta temática del desarrollo integral de los adolescentes como la Declaración Mundial sobre Educación Superior en el Siglo XXI de la UNESCO (1998, citada en Fernández Mojica y Barradas Alarcón, 2014). En su estudio diagnóstico de la universidad se destaca el vacío espiritual. El documento propone el desarrollo integral en tres dimensiones: formación intelectual, formación social y formación humana: afirman que esta última incluye el desarrollo espiritual. Fernández Mojica y Barradas Alarcón, al analizar planes de estudios de una universidad mexicana, detectaron que "en ninguna experiencia educativa se aborda la dimensión espiritual" (p. 65).

Esta preocupación debe servir como un espejo y ser atendida por la 


\section{GARCÍA FIGUEROA}

administración de las escuelas privadas de El Salvador, para que sus docentes efectúen una integración de la fe en la formación de los estudiantes.

Por su parte, Rosas de León y Labarca Reverol (2016) encontraron que la espiritualidad ayuda a afrontar la cultura de la violencia por medio de la interiorización de valores, motivación y comportamiento, más allá de asistir a una iglesia en particular; es efectivo el ambiente religioso como una manera de vivir y superar los problemas, a la vez que promueve el desarrollo integral y la cultura de la paz.

Algunos estudios correlacionales previos sobre la percepción de la calidad del ambiente y el grado de calidad de la conducta percibida (Cerna Vanegas y Meza Escobar, 2009; Jesse Rock, 2011) mostraron que las dos variables que se relacionan con mayor grado de influencia sobre la conducta de los estudiantes de tercer ciclo son el ambiente familiar y el ambiente religioso. Un estudio reciente (Díaz Castellar, 2019) sobre la correlación entre ambiente familiar y práctica de valores en adolescentes con similares edades y distribución de género mostró que los hogares con menor apoyo de los padres presentan manifestaciones de mayor hostilidad y problemas de relaciones sociales entre los adolescentes que se aferran a medios materiales como sustitución a la carencia de afecto. En cambio, quienes reciben mayor apoyo de los padres presentan mayores prácticas de valores morales, religiosos y sociales, como evidencia de que el ambiente familiar es predictor de la conducta para los adolescentes.

El ambiente familiar tiene una influencia significativa sobre la adaptación social del adolescente al ingresar al bachillerato, principalmente entre los estudiantes varones, y especialmente en la dimensión cohesión familiar (Verdugo Lucero et al., 2014).

En síntesis, los resultados del estudio permiten inferir que el grado de calidad del ambiente general, que incluye la influencia del ambiente espiritual, familiar y del vecindario se refleja en la calidad de la conducta de los estudiantes de los colegios participantes.

\section{Referencias}

Arce, R., Fariña, F. y Vázquez, M. J. (2011). Grado de competencia social y comportamientos antisociales, delictivos y no delictivos en adolescentes. Revista Latinoamericana de Psicología, 43(3), 473-486. https://doi.org/10.14349/rlp.v43i3.487

Bachman, K. (2007). The relationship between divorce, interparental conflict and adolescent social skills (Publicación N³273260) [Tesis doctoral, Alliant International University]. ProQuest Dissertations and Theses Global.

Boarini, L. M. (2013). Indisciplina escolar: uma construção coletiva. Psicologia Escolar e Educacional, 17(1), 123-131. https://doi.org/10.1590/S1413-85572013000100013

Bustos Correa, C., Herrera, M. O. y Mathiesen, M. E. (2001). Calidad del ambiente del hogar: inventario HOME como un instrumento de medición. Estudios Pedagógicos, 27, 7-22. https://doi.org/10.4067/ S0718-07052001000100001

Cantón-Cortés, D., Cortés Arboleda, M. y Cantón Duarte, J. (2010). Experiencias traumáticas, ambiente familiar y ajuste psicológico. INFAD 1(1), 363-369. http://infad.eu/RevistaINFAD/2010/n1/INFAD _010122_363-369.pdf

Carranza, P., Sgreccia, N., Quijano, T., Goin, M. y Chrestia, M. S. (2017). Ambientes de aprendizaje y proyectos escolares con la comunidad. Revista Latinoamericana de Etnomatemática, 10(1), 50-61. https://www.revista.etnomatematica.org/index.php/RevLatEm/article/view/324 


\section{CALIDAD DEL AMBIENTE Y CALIDAD DE LA CONDUCTA}

Cerna Vanegas, C. S. y Meza Escobar, M. R. (2009). Ambiente escolar, familiar, social y religioso y calidad de la conducta de los estudiantes de colegios secundarios. Revista Internacional de Estudios en Educación, 9(1), 34-48. https://doi.org/10.37354/riee.2009.088

Dias Vieira, A. C. y Abu-Jamra Zornig, S. M. (2015). Ambiente violento, infância perdida? Revista Latinoamericana de Psicopatologia Fundamental, 18(1), 88-101. https://doi.org/10.1590/1415$4714.2015 \mathrm{v} 18 \mathrm{n} 1 \mathrm{p} 88.7$

Díaz Castellar, E. J. (2019). Condición socioeconómica y ambiente familiar relacionado con la práctica de valores en estudiantes en la institución educativa adventista de Cartagena, Colombia [Tesis de maestría, Universidad de Montemorelos]. DSpace. http://dspace.biblioteca.um.edu.mx/xmlui/ handle/20.500.11972/1104

Driskell, R. L. y Lyon, L. (2011). Assessing the role of religious belief on secular and spiritual behaviors. Review of Religious Research, 52(4), 386-404.

Fernández Mojica, N. y Barradas Alarcón, M. E. (2014). Vacío espiritual en experiencias educativas. Revista de Comunicación de la SEECI, 18(Número extraordinario), 59-66. https://doi.org/10.15198/ seeci.2014.35E.59-66

Foà, C., Brugman, D. y Mancini, T. (2012). School moral atmosphere and normative orientation to explain aggressive and transgressive behaviours at secondary school. Journal of Moral Education, 41(1), 1-22. https://doi.org/10.1080/03057240.2011.652520

Francis, L., Siôn, T. y Village, A. (2014). Measuring the contribution of independent Christian secondary schools to students' religious, personal, and social values. Journal of Research on Christian Education, 23(1), 29-55. https://doi.org/10.1080/10656219.2014.882723

Grier, L. y Gudiel, W. (2011). Can religious beliefs combat negative peer influence during adolescence? Mental Health, Religion \& Culture, 14(10), 983-997. https://doi.org/10.1080/13674676.2010.542452

Guerra Vio, C., Vargas Castro, J., Castro Arancibia, L., Plaza Villareal, H. y Barrera Montes, P. (2012). Percepción del clima escolar en estudiantes de enseñanza media de Valparaíso de colegios municipales, particulares subvencionados y particulares. Estudios Pedagógicos, 38(2), 103-115. https://doi.org/10.4067/S0718-07052012000200007

Infante, L., Hierrezuelo, L., García, B., Sánchez, A., De la Morena, M. L., Muñoz, A. y Trianes, M. V. (2003). Evaluación de actitudes violentas y clima escolar en situaciones de agresividad en alumnado de secundaria. Psicologia, Saúde \& Doenças, 4(2), 277-286. https://www.sp-ps.pt/site/jr/4

Jesse Rook, L. R. (2011). Factores del ambiente general predictores de la conducta de alumnos de colegios adventistas de Belice [Tesis de maestría, Universidad de Montemorelos]. DSpace. http:// dspace.biblioteca.um.edu.mx/xmlui/handle/20.500.11972/85

Jiménez, T. I., Estévez, E. y Murgui, S. (2014). Ambiente comunitario y actitud hacia la autoridad: relaciones con la calidad de las relaciones familiares y con la agresión hacia los iguales en adolescentes. Anales de Psicología, 30(3), 1086-1095. https://doi.org/10.6018/analesps.30.3.160041

Johnson, S. L., Burke, J. G. y Gielen, A. C. (2012). Urban students' perceptions of the school environment's influence on school violence. Children and Schools, 34(2), 92-102. https://doi. org/10.1093/cs/cds016

Karimi Koma, M. (2014). Comparison of students' social behaviors under the supervision of school principals graduates educational administration or related fields and other students. International Journal of Social Sciences \& Education, 4(Número especial), 138-147. http://ijsse.com/sites/default/ files/issues/2014/v4-i4-2014/Paper-17.pdf

Loona, M. I. y Kamal, A. (2012). Role of perceived parenting styles and familial factors in prediction of teacher-report childhood behavior problems. Journal of Behavioural Sciences, 22(3), 49-69. http:// pu.edu.pk/images/journal/doap/PDF-FILES/Abstract\%204_v22_3_2012.pdf 


\section{GARCÍA FIGUEROA}

Mayorga Muñoz, C., Godoy Bello, M. P., Riquelme Sandoval, S., Ketterer Romero, L. y Gálvez Nieto, J. L. (2016). Relación entre problemas de conducta en adolescentes y conflicto interparental en familias intactas y monoparentales. Revista Colombiana de Psicología, 25(1), 107-122. https://doi. org/10.15446/rcp.v25n1.48705

Moreno Ruiz, D., Estévez López, E., Murgui Pérez, S. y Musitu Ochoa, G. (2009). Relación entre el clima familiar y el clima escolar: el rol de la empatía, la actitud hacia la autoridad y la conducta, violenta en la adolescencia. International Journal of Psychology and Psychological Therapy, 9(1), 123-136. https://www.ijpsy.com/volumen9/num1/226/relacin-entre-el-clima-familiar-y-el-clima-ES.pdf

Papšová, M., Valihorová, M. y Nábělková, E. (2012). Exploring the moderating effect of field dependence-independence on relationship between pupil's school satisfaction and social classroom atmosphere. Studia Psychologica, 54(2), 125-136. https://www.studiapsychologica.com/uploads/ PAPSOVA_SP_02_vol.54_2012_pp.125-136.pdf

Petts, R. J. (2011). Is urban fathers' religion important for their children's behavior? Review of Religious Research, 53(2), 183-206. https//doi.org/10.1007/s13644-011-0010-9

Pillon, S. C., dos Santos, M. A., de Souza Gonçalves, A. M. y de Araújo, K. M. (2011). Uso de álcool e espiritualidade entre estudantes de enfermagem. Revista da Escola de Enfermagem da USP, 45(1), 100-107. https://doi.org/10.1590/S0080-62342011000100014

Pitsoane, E. M. y Gasa, V. G. (2018). The role of father-son relationship in behavioural and emotional development of adolescent boys. Gender \& Behaviour, 16(1), 10748-10757. https://www.ajol.info/ index.php/gab/article/view/175230

Quiroz del Valle, N., Villatoro Velázquez, J. A., Juárez García, F., Gutiérrez López, M. L., Amador Buenabad, N. G. y Medina-Mora Icaza, M. E. (2007). La familia y el maltrato como factores de riesgo de conducta antisocial. Salud Mental, 30(4), 47-54. http://revistasaludmental.mx/index.php/ salud_mental/article/view/1179/1177

Rivero, N. y Martínez-Pampliega, A. (2010). Adaptación cultural del instrumento "Patrones de Comunicación Familiar- R". European Journal of Education and Psychology, 3(1), 141-153. https:// doi.org/10.30552/ejep.v3i1.35

Rosas de León, Y. E. y Labarca Reverol, C. (2016). Violencia, espiritualidad y resiliencia en estudiantes de la unidad educativa arquidiocesana "bicentenario del natalicio del libertador". Telos: Revista de Estudios Interdisciplinarios en Ciencias Sociales, 18(2), 302-317. https://www.redalyc.org/articulo .oa? $\mathrm{id}=99345727009$

Schottenbauer, M. A., Spernak, S. M. y Hellstrom, I. (2007). Relationship between family religious behaviors and child well-being among third-grade children. Mental Health, Religion \& Culture, 10(2), 191-198. https://doi.org/10.1080/13674670600847394

Slattery, T. L. y Meyers, S. A. (2014). Contextual predictors of adolescent antisocial behavior: The developmental influence of family, peer, and neighborhood factors. Child \& Adolescent Social Work Journal, 31(1), 39-59. https://doi.org/10.1007/s10560-013-0309-1

Valdemoros-San-Emeterio, M., Sanz-Arazuri, E. y Ponce-de-León-Elizondo, A. (2017). Ocio digital y ambiente familiar en estudiantes de postobligatoria. Comunicar, 25(50), 99-107. https://doi.org/10.3916/C50-2017-09

Verdugo Lucero, J. C., Arguelles Barajas, J., Guzmán Muñiz, J., Márquez González, C., Montes Delgado, R. y Uribe Alvarado, I. (2014). Influencia del clima familiar en el proceso de adaptación social del adolescente. Psicología desde el Caribe, 31(2), 207-222. https://doi.org/10.14482/psdc.31.2.6127

Recibido: 21 de mayo de 2020

Revisado: 20 de junio de 2020

Aprobado: 3 de julio de 2020 